DOI: 10.3901/JME.2019.02.214

\title{
抗磁悬浮研究综述
}

\author{
徐园平 ${ }^{1,2}$ 周 瑾 ${ }^{1}$ 金超武 $^{1}$ BLEULER Hannes ${ }^{2}$ \\ (1. 南京航空航天大学机电学院 南京 210016; \\ 2. 瑞士洛桑联邦理工学院(EPFL) 洛桑 1015 瑞士)
}

\begin{abstract}
摘要: 抗磁悬浮技术利用物质的抗磁特性, 无需外界能量输入即可实现常温被动静态稳定悬浮, 获得众多学者的关注。尤其 是近 30 年来, 随着微细制造与强磁场技术的发展, 抗磁悬浮相关研究持续增加。目前, 国外众多研究机构基于抗磁悬浮原 理已经开展了较为丰富的相关研究工作, 但国内在该领域的研究成果相对较少, 研究内容较为单一。针对国内该领域这一研 究现状, 从抗磁悬浮机理、抗磁悬浮特点及抗磁悬浮相关应用研究成果进行全面的综述。提出抗磁悬浮不仅具备无磨损、无 需润滑、低能耗等优点外, 还具有常温被动静态稳定悬浮、微纳米尺度应用、低刚度等特点; 指出该技术在微纳旋转机械、 振动能量捕获、高灵敏度传感器、生物细胞/微粒子操纵等领域具有非常广阔的应用前景。为国内学者开展相关抗磁悬浮研究 提供了综述资料。
\end{abstract}

关键词: 抗磁悬浮; 机理; 特点; 应用; 综述

中图分类号: $\mathrm{T} 1$

\section{Diamagnetic Levitation: A Review}

\author{
XU Yuanping ${ }^{1,2}$ ZHOU Jin $^{1} \quad$ JIN Chaowu $^{1} \quad$ BLEULER Hannes ${ }^{2}$ \\ (1. College of Mechanical and Electrical Engineering, \\ Nanjing University of Aeronautics and Astronautics, Nanjing 210016; \\ 2. École Polytechnique Fédérale de Lausanne (EPFL), Lausanne 1015, Switzerland)
}

\begin{abstract}
With the diamagnetic material property, diamagnetic levitation possesses the special advantages of passive static and stable levitation at room temperature, which has attracted many scholars. Especially in the recent three decades, with the development of strong magnetic field equipment and micro technology, increasing publications on diamagnetic levitation research are appearing. Many foreign researches have done the relative investigations; however, the domestic researches in this field are relatively few and narrow. Aimed at this situation, a review of diamagnetic levitation technology including diamagnetic theory, diamagnetic characteristics and relative application research works is presented. The diamagnetic levitation technology not only possesses free-contact, no-lubrication and low energy consumption merits, but also permits advantages such as passive static and stable levitation at room temperature, micro-nano application and low supporting stiffness, which may possess potential and promising application ranging from micro rotating machinery, energy harvesting, high sensitive MEMS-sensor to cell/particle manipulation. A review of diamagnetic levitation is provided, which is helpful for the domestic researchers.
\end{abstract}

Key words: diamagnetic levitation; theory; characteristics; application; review

\section{0 前言}

自然界的物质在外加磁场的作用下会产生附加 磁场，表现出磁性(即磁化现象)。根据磁介质中所 产生的附加磁场与外磁场方向, 弱磁性物质可分为 顺磁性和抗磁性。顺磁性物质中产生的附加磁场与

20170706 收到初稿, 20180801 收到修改稿
外磁场方向相同; 而抗磁性物质相反。当抗磁性物 质处于外加磁场中时, 由于附加磁场与外磁场方向 相反, 会产生微弱的斥力, 即抗磁力。利用抗磁性 物质的抗磁性使其在磁场中稳定悬浮的技术称为抗 磁悬浮技术。

物质的抗磁性最初由 BRUGMANS 在 1778 年 发现。1845 年 FARADAY 将这种特性命名为抗磁性 (Diamagnetism) ${ }^{[1]}$ 。THOMSON 在 1847 年理论预测 了抗磁悬浮的可行性 ${ }^{[2]}$, 但由于抗磁力自身非常微 
弱, 研究人员当时并未对抗磁悬浮进行深入的研究, 甚至持怀疑态度。直到近 30 年来, 随着微细制造与 强磁场技术的发展, 抗磁悬浮相关研究及应用才开 始兴起。

抗磁悬浮技术有以下两个典型的研究成果 ${ }^{[3-5]}$ :

(1) 1997 年, BERRY 和 GEIM 利用 $16 \mathrm{~T}$ 的强磁场, 首次实现了活体青蛙的稳定悬浮, 如图 1a 所示;

(2) 1999 年, GEIM 带领的研究小组利用人体的抗磁 特性, 在距离强磁场 $2.5 \mathrm{~m}$ 处通过手指稳定悬浮 $\mathrm{NdFeB}$ 永磁体, 如图 1b 所示。其研究成果发表于 《Nature》期刊。

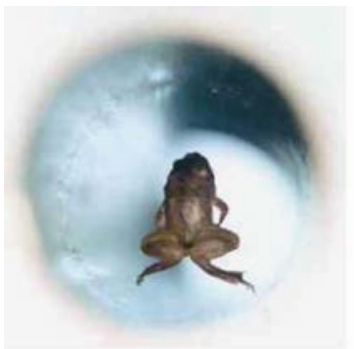

(a) 活体青蛙的悬浮

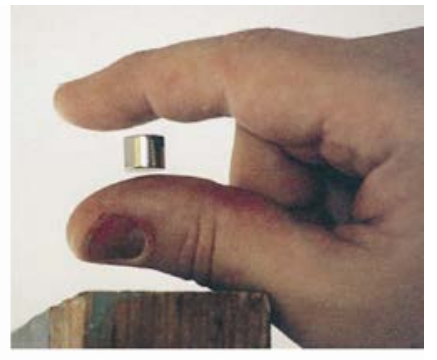

(b) 手指稳定悬浮 $\mathrm{NdFeB}$ 永磁体
图 1 基于抗磁悬浮技术的典型研究成果

\section{1 抗磁悬浮原理}

\section{1 抗磁效应}

自然界的物质在外加磁场的作用下会产生附加 磁场。根据磁介质中所产生的附加磁场与外磁场方 向, 弱磁性物质可分为顺磁性和抗磁性。事实上大 多数被认为无磁性的物质都具有极弱抗磁性, 例如 水、铜和蛋白质等。当抗磁性物质处于外加磁场中 时, 由于附加磁场与外磁场方向相反, 会产生微弱 的斥力, 即抗磁力。在外加磁场磁通密度(磁感应强 度) $B$ 下, 抗磁性物质单位体积所受到的抗磁力 $f$ 可 以表示为

$$
f=M_{d} \cdot \nabla(B)
$$

式中, $M_{d}$ 为磁性物质的磁化强度。磁通密度 $B$ 与 磁场强度 $H$ 之间的关系可以表示为

$$
H=\frac{B}{\mu_{0}}-M_{d}
$$

式中, $\mu_{0}=4 \pi \times 10^{-7} \mathrm{~N} \cdot \mathrm{A}^{-1}$ 是真空磁导率。磁化强度 $M_{d}$ 与磁场强度 $H$ 之间存在以下关系

$$
M_{d}=\chi_{m} H
$$

式中, $\chi_{m}$ 为磁化率。顺磁性物质的磁化率 $\chi_{m}>0$; 抗磁性物质的磁化率 $\chi_{m}<0$ 。表 1 列举了常见的抗 磁性物质的磁化率。
表 1 常见抗磁物质的磁化率

\begin{tabular}{cc}
\hline 物质名称 & 磁化率 $\chi_{m} / \times 10^{-6}$ \\
\hline 水 & -9.0 \\
铋 & -160 \\
热解石墨 $\perp$ & -450 \\
热解石墨 $\|$ & -85 \\
超导 & $-10^{6}$ \\
血红蛋白 & -3.4 \\
DNA & -5.0 \\
\hline
\end{tabular}

对于抗磁性物质, 由于磁化率 $\chi_{m}$ 非常微弱, 所 以联立式(2)、(3), $M_{d}$ 与 $B$ 之间的关系可表示为

$$
M_{d}=\frac{\chi_{m}}{\left(1+\chi_{m}\right) \mu_{0}} B \approx \frac{\chi_{m}}{\mu_{0}} B
$$

由式(1)、(4), 抗磁性物质单位体积所受到的抗 磁力可表示为

$$
f=\frac{\chi_{m}}{\mu_{0}} B \cdot \nabla(B)
$$

从表 1 中可以看出, 由于抗磁性物质的磁化率 $\chi_{m}<0$, 所以抗磁性物质与外加磁场之间会产生排 斥力, 使得抗磁性物质由高磁通密度向低磁通密度 方向运动, 如图 2 所示。

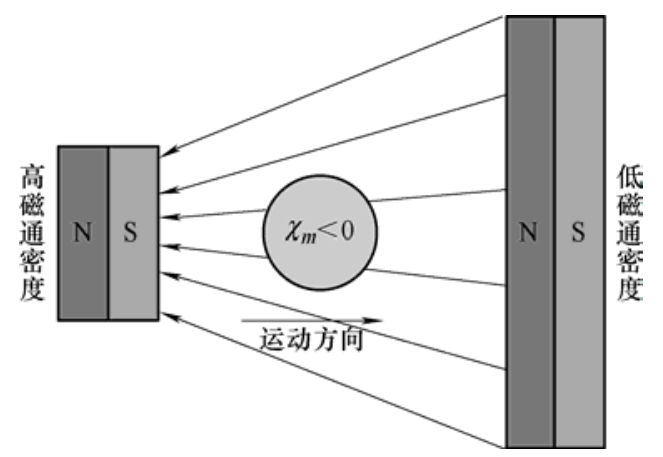

图 2 抗磁效应示意图

式(5)为真空环境下无介质的磁化效应时产生 的抗磁力, 若考虑介质的磁化效应, 则抗磁性物质 单位体积所受到的抗磁力可表示为

$$
f=\frac{\chi_{m}-\chi_{i}}{\mu_{0}} B \cdot \nabla(B)
$$

式中, $\chi_{i}$ 为介质的磁化率。由式(6)可知, 在外磁场 环境下, 将抗磁性物质置于顺磁性介质中, 例如 $\mathrm{MnCl}_{2}$ 溶液、 $\mathrm{NaCl}-\mathrm{KCl}$ 混合溶液或某些顺磁性气体, 顺磁性介质会受到外磁场磁化, 这时抗磁性物质除 受磁场对它的排斥力外, 还受到介质对抗磁性物质 产生的抗磁力和顺磁介质提供的浮力, 会极大地提 高抗磁效应, 即基于磁阿基米德效应的抗磁悬浮, 由 IKEZOE 等于 1998 年在《Nature》期刊上提出并 实现。由式(6)也可以得出, 弱顺磁性物质置于强顺 
磁性介质中时, 由于 $\left(\chi_{m}-\chi_{i}\right)<0$, 也可产生抗磁力, 实现悬浮功能。

\section{2 抗磁悬浮原理}

EARNSHAW $^{[6]}$ 定理指出, 仅靠永磁体之间的作 用力或者永磁体与顺磁性物质之间的作用力是无法 实现被动静态稳定悬浮。但不同于永磁体之间或者 永磁体与顺磁性物质之间的作用力, 抗磁性物质依 靠抗磁力, 可实现被动静态稳定悬浮 ${ }^{[7-8]}$ 。

不考虑介质的磁化效应, 式(5)为抗磁性物质单 位体积所受到的磁力, 所以抗磁性物质所受合力为 体积内的积分

$$
F=\frac{\chi_{m}}{2 \mu_{0}} \int_{v} \nabla\left(B^{2}\right) \mathrm{d} V
$$

当磁场的磁通密度呈 “凹型” 分布时, 即有可 能实现被动静态稳定悬浮。通过对永磁体合理的排 布, 例如磁极反向排布、“Halbach” 排布等, 都可 以构造出磁通密度呈凹型分布的磁场, 即凹型磁势 能吽。图 3 为用于抗磁悬浮的两种常用的永磁体排 布方式。

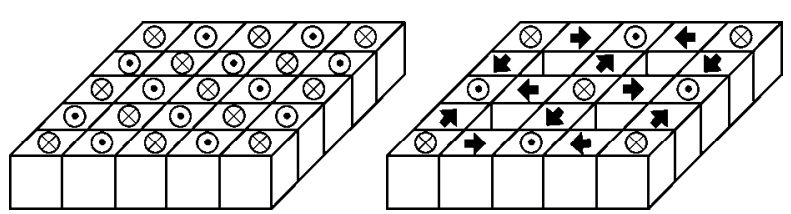

(a) 磁极反向排布

(b) Halbach 排布

图 3 可实现稳定抗磁悬浮的永磁体排布方式

图 4 是本文作者所在研究组利用环形永磁体与 圆柱体永磁体正反磁极排布, 实现了热解石墨片的 稳定悬浮。图 5 为根据文献[9]中的永磁体建模方法, 建模求解获得的永磁体上方 $600 \mu \mathrm{m}$ 处, 坚直方向永 磁体势能分布图 $\left(B^{2}\right)$ 。从图中可以看出, 此时永磁 体势能呈凹型分布。即中间磁场势能低, 四周磁场 势能高, 满足抗磁稳定悬浮的要求。

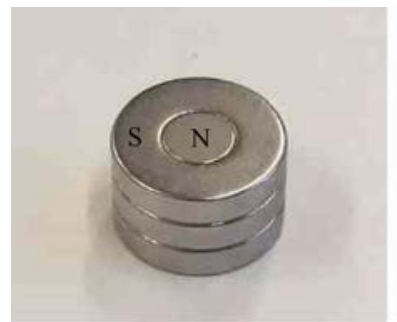

(a) 正反磁极排布永磁体

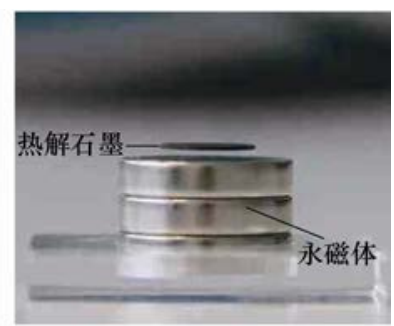

(b) 热解石墨片的稳定悬浮
图 4 抗磁悬浮试验图

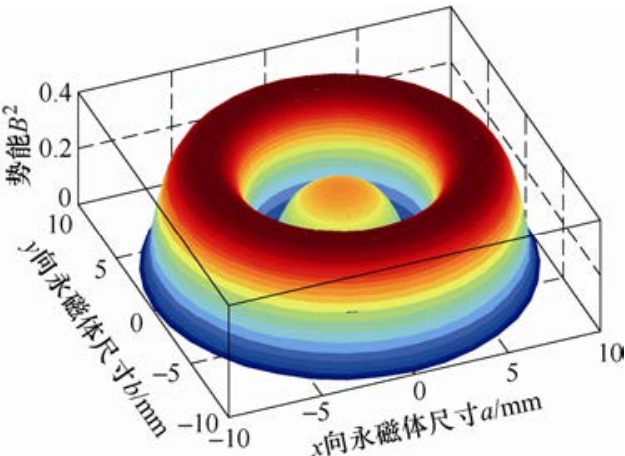

图 5 永磁体上方 $600 \mu \mathrm{m}$ 处垂直方向永磁体 势能 $\left(B^{2}\right)$ 分布图

为磁悬浮技术的一个分支, 抗磁悬浮除具备上述优 点外, 还有以下几方面特点。

\section{1 低刚度弱抗磁效应}

除超导材料外的抗磁材料在大尺度下抗磁效应 非常微弱, 相关研究表明抗磁悬浮支承刚度在 10 $\mathrm{N} / \mathrm{m}$ 范围 ${ }^{[10]}$ 。尽管抗磁悬浮支承刚度较低, 但其低 刚度的特点可以用于纳牛顿级力传感器、低频加速 度传感器以及低频振动能量的收集。

\section{2 微纳尺度应用}

主动磁悬浮技术在宏观领域已实现广泛应用。 但在 $\mathrm{mm}$ 尺度及其以下的微纳系统中难以得到应 用，主要有以下两点原因。

（1）由于主动磁悬浮结构本身含有铁芯、线圈 绕组等部件, 所以难以在 $\mathrm{mm}$ 尺度及其以下的微纳 系统中实现。

(2) 在微纳磁悬浮系统中，相对于被悬浮物体, 气隙变得相对很大。此时主动磁悬浮的漏磁将会随 着尺度减小而急剧增大。而抗磁悬浮技术可以弥补 上述缺点。虽然抗磁性物质的抗磁力微弱, 但抗磁 力与物质重力之比将随着系统整体尺寸的减小而增 大, 因而抗磁悬浮原理适宜于微器件中 ${ }^{[11-12] 。}$

\section{3 被动静态稳定悬浮}

EARNSHAW $^{[6]}$ 定理指出如果物质所受到的作 用力是与距离的平方成反比时, 这些物质都无法实 现被动静态稳定悬浮。因此无论静电场、静磁场和 重力场怎样组合都无法实现被动静态稳定悬浮。但 是抗磁效应不同于 EARNSHAW 定理。通过构建出 具有凹型的磁势能阱, 可以实现常温下抗磁悬浮物 质被动静态稳定悬浮, 完全无需外界能量。

\section{3 抗磁悬浮相关研究}

目前, 基于抗磁悬浮原理的应用研究主要集中 在微纳旋转机械、振动能量捕获、高灵敏度传感器、 生物细胞/微粒子操纵等领域。 


\section{1 抗磁悬浮微纳旋转机械}

在抗磁悬浮微纳旋转机械方面, 国内外相关研 究处于起步阶段, 大部分研究侧重于抗磁悬浮的物 理特性研究, 少数研究实现了低速旋转功能。

CANSIZ 等 ${ }^{[13-14]}$ 设计了如图 6 所示的试验装置。 通过利用抗磁性物质铋, 实现永磁体的稳定悬浮。 然后借助氮喷射推动抗磁转子高速旋转, 在转子自 由降速阶段, 研究了抗磁轴承的动态特性。郑州大 学 SU 等 ${ }^{[15-16]}$ 将高定向热解石墨(Highly oriented pyrolytic graphite)作为转子, 借助氮喷射达到了 500 $\mathrm{r} / \mathrm{min}$ 。上述研究均采用外部气体来推动抗磁悬浮转 子旋转, 并未实现类似于电机驱动的主动可控旋转 功能。CHEN 等 ${ }^{[17-18]}$ 对抗磁轴承的稳定性与浴流效 应从理论上进行了分析。

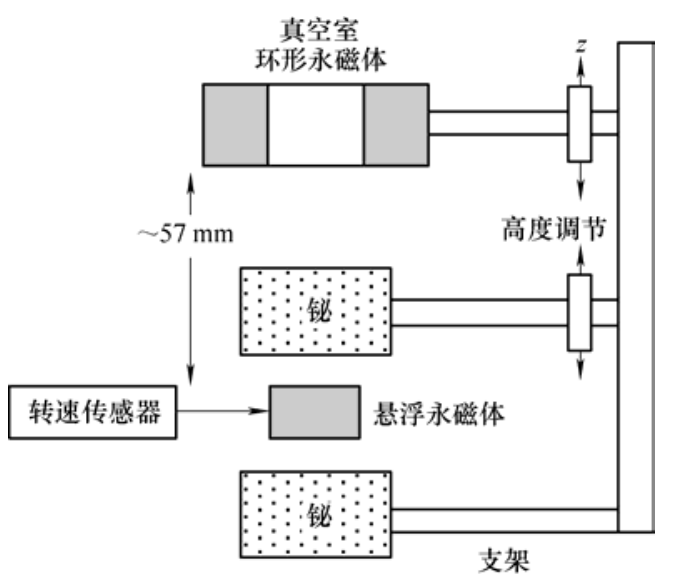

图 6 抗磁悬浮转子动态特性测试装置

针对旋转功能的实现, 瑞士洛桑联邦理工学院 BLEULER 等 ${ }^{[19]}$ 提出了抗磁悬浮与静电驱动结合的 方案, 但此方案并未实施。上海交通大学微纳米研 究中心 LIU 等 ${ }^{[20-21]}$ 研制了基于可变电容驱动原理抗 磁悬浮转子系统(图 7), 实现了低速旋转功能 (10 $\mathrm{r} / \mathrm{min}$ ); 并在后续的研究中提出了基于可变磁场原理 的旋转方法 ${ }^{[22]}$ 。

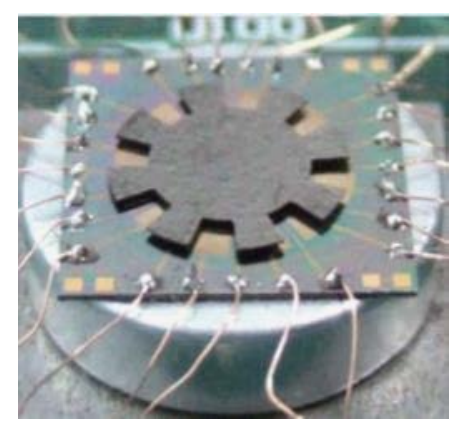

图 7 基于可变电容驱动的抗磁悬浮转子

本文课题组研制出了基于高压静电驱动原理抗

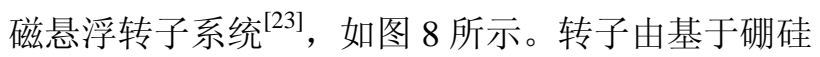
酸盐玻璃与热解石墨组成, 静电驱动电机定子位于
转子上侧。在 $\pm 300 \mathrm{~V}$ 的三相静电场的驱动下, 试 验观测到最高 $140 \mathrm{r} / \mathrm{min}$ 的转速, 并在后续的研究 中, 实现了 $300 \mathrm{r} / \mathrm{min}$ 的转速。 (a) 转子稳定悬浮

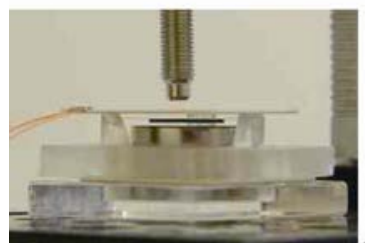

(c) 装置实物图

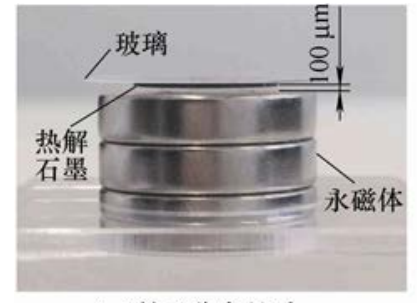

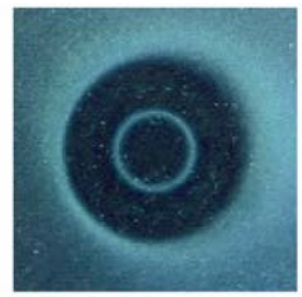

(b) 磁场观察膜下磁场分布

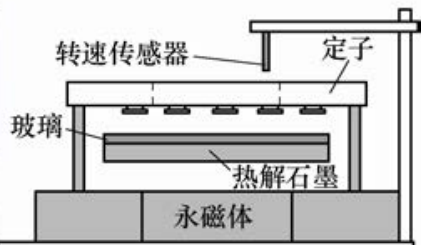

(d) 装置示意图
图 8 高压静电驱动抗磁悬浮转子

目前抗磁悬浮微纳旋转机械相关已有研究成果 大多侧重于抗磁悬浮轴承的物理动态特性研究, 而 针对转子旋转功能的实现的相关研究成果相对较 少, 且转速相对较低。由于高转速下微纳旋转机械 的性能将会有极大提升, 且抗磁悬浮具有无摩擦、 被动稳定悬浮的特点, 所以可实现高速功能抗磁 悬浮的微纳旋转机械是目前研究的热点, 已经引 起众多学者的关注。例如, CIRRAN 等 ${ }^{[24]} 2015$ 年提出了将抗磁悬浮陀螺作为微型卫星姿态控制 的计划。

\section{2 抗磁悬浮振动能量捕获}

抗磁悬浮力的低刚度特性降低了整个悬浮系统 共振频率，非常适宜于用于低频振动能量收集。与 此同时, 其微纳尺度应用的特点, 适宜于研制微小 的能量捕获装置, 因此被相关学者关注。

北卡罗莱纳州立大学 YUAN 教授研究组自 2011 年起对基于抗磁特性的能源收集方法开展了 相关研究。其研究团队先后研制出了水平抗磁悬浮 能量捕获装置(图 9) 与坚直抗磁悬浮能量捕获装置 (图 10), 并开展了理论与试验研究 ${ }^{[25-29]}$ 。上海交通 大学 ZHANG 等 ${ }^{[30]}$ 借助抗磁效应, 设计制作了一种 双稳态振动能量捕获装置, 试验研究表明该能量捕 获装置在极低的振动频率下 $(<5 \mathrm{~Hz})$, 可以高效地工 作。意大利都灵理工、国内郑州大学也对抗磁悬浮 能量捕获领域开展了相关类似研究 ${ }^{[31-34]}$ 。上述抗磁 悬浮振动能量捕获装置, 永磁体悬浮与两片固定的 热解石墨薄片之间。热解石墨片上布置有线圈, 当 外界振动引起永磁体与线圈产生相对运动时, 线圈 会切割磁感线产生电动势, 将动能转换为电能。然 
而上述装置为了弥补弱抗磁效应导致永磁体重力大 于抗磁力无法悬浮的问题, 均需要提升永磁体 (Lifting magnetics) 来补偿重力影响。这导致整个 系统结构更加复杂, 并只有精密地调节提升永磁体 的位置才能实现稳定悬浮的功能。

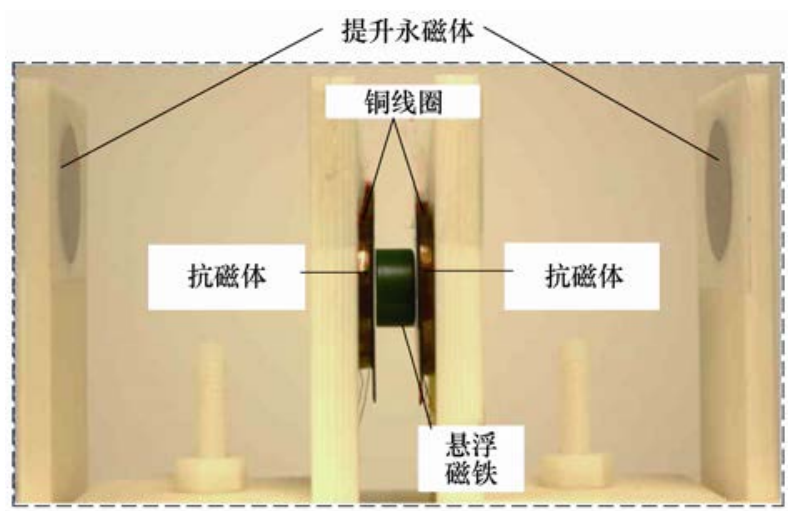

图 9 基于浴流效应的水平抗磁悬浮能量捕获装置

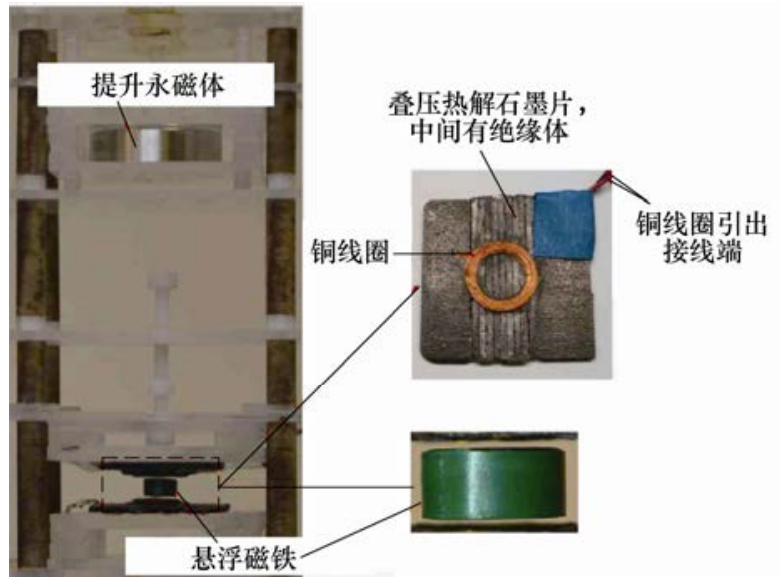

图 10 基于浴流效应的坚直抗磁悬浮能量捕获装置

在能量捕获方面的研究, 另一项代表性的研究 成果是日本 KOBAYASHI 等 ${ }^{[35]}$ 在 2012 年利用光热 作用下, 热解石墨的磁化率会发生变化的特性, 借 助太阳光, 驱动悬浮的热解石墨转子达到 $200 \mathrm{r} / \mathrm{min}$ 转速。这一研究提供了一条新的太阳能收集转换方 法。图 11 为抗磁悬浮热解石墨转子在光束驱动下进 行旋转。
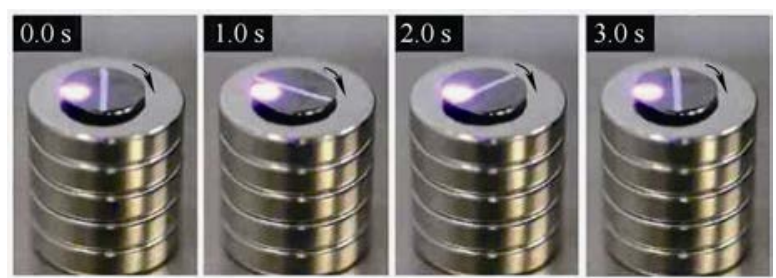

图 11 光驱动抗磁悬浮热解石墨转子

\section{3 抗磁悬浮高灵敏度传感器}

抗磁力的无摩擦、低刚度特性, 适宜于高灵敏 度传感器。1968 年, SIMON 等 ${ }^{[36]}$ 利用热解石墨悬
浮体在永磁场中稳定悬浮原理制作了如图 12 所示 的倾角测试仪, 其测量范围为 $\pm 1.5^{\circ}$, 分辨率优于 $\pm 0.1^{\prime \prime}$ 。

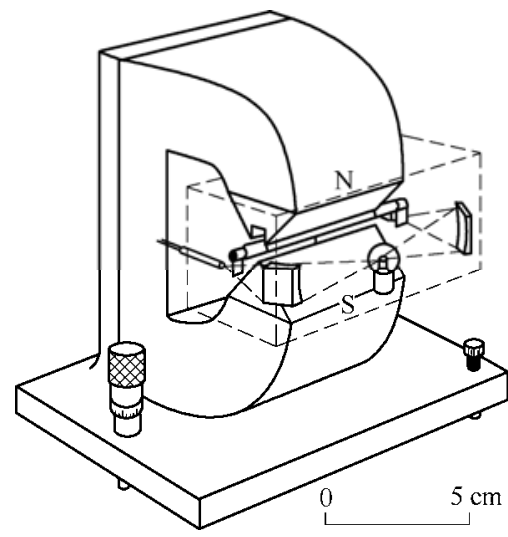

图 12 抗磁悬浮倾角测试仪

2001 年, GEIM 等 ${ }^{[37]}$ 在强磁场中悬浮直径 $2 \mathrm{~mm}$ 塑料小球, 并使用气流将小球加速旋转至 $20 \mathrm{~Hz}$ 。 此时, 通过肉眼观察高速小球的方位即可验证地球 的自转速度。其试验示意如图 13 所示, 文中指出抗 磁悬浮有望用于超高精度陀螺。

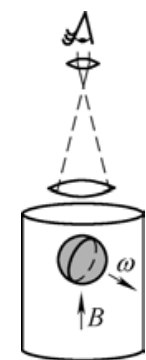

图 13 悬浮塑料小球试验示意图

2003 年, PIAT 等 ${ }^{[38]}$ 利用抗磁稳定永磁悬浮设 计了纳牛顿级的力传感器, 获得的精度达到 $19 \mathrm{nN}$, 并且将其应用于微米级粒子的操纵。在后续研究 中, 该研究组设计了基于差分抗磁悬浮原理的纳牛 顿级传感器, 如图 14 所示, 用于弥补外界环境的

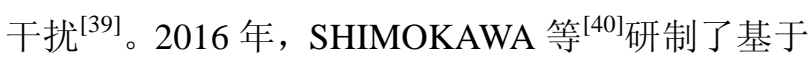
抗磁悬浮的气体粘性测量系统, 如图 15 所示。转 子由热解石墨片与铝片黏合组成, 悬浮在环形永磁 体上方。整个悬浮系统置于密闭的被测气体中。在 转子上方, 电机带动片状方形组合永磁体旋转, 产 生旋转磁场, 驱动转子转动。借助抗磁悬浮特点, 该装置实现了在 $\mu \mathrm{Pa} \cdot \mathrm{s}$ 范围内气体粘性的精确测 量。GUNAWAN 等 ${ }^{[41]}$ 利用 2 个径向充磁的圆柱形 永磁体, 实现了对圆柱形热解石墨的稳定悬浮(图 16), 并提出了基于该原理的高灵敏霍尔传感器方 案。CAO 等 ${ }^{[22-44]}$ 基于抗磁悬浮原理, 实现了高密 度物质的密度测量。 


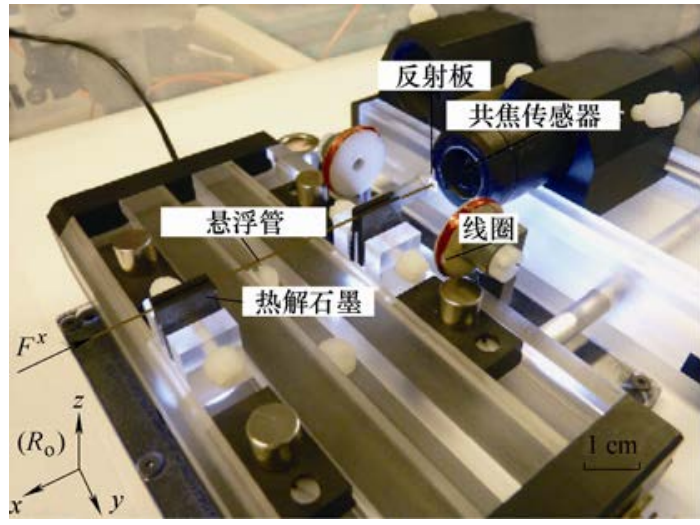

图 14 基于抗磁悬浮原理的纳牛顿级传感器

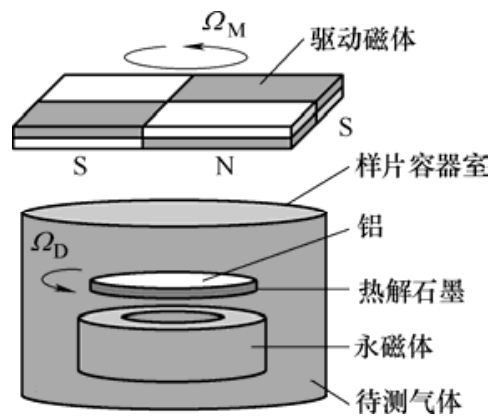

图 15 基于抗磁悬浮的气体粘性测量装置示意图

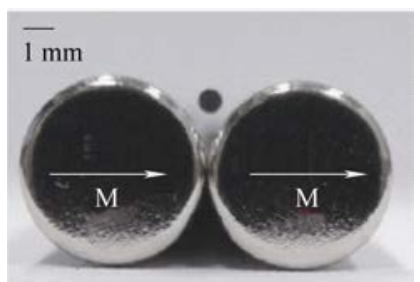

图 16 圆柱形热解石墨稳定悬浮

\section{4 抗磁生物细胞/微粒子操纵}

生命科学技术研究需要借助微操纵对单个或数 个生物活性粒子实施捕获、迁移、分离等。微操纵 方式可以分为接触式和非接触式生物微操纵。接触 式施力, 容易损坏生物粒子的活性, 所以越来越多 的研究者探索非接触式生物粒子的操纵研究。由于 大多数生物粒子自身具备的抗磁性, 所以作为非接 触式操纵方法中的一员, 基于抗磁效应的微粒子操 纵具有广阔的应用前景。

IKEZOE 等 ${ }^{[45]} 1998$ 年首次在 《Nature》期刊上提 出抗磁悬浮磁阿基米德原理, 利用 $10 \mathrm{~T}$ 的磁场强度, 即对水滴实现了悬浮。后续研究中, 其团队利用抗 磁悬浮磁阿基米德原理, 实现了对微弱的磁性粒子 及生物粒子的分离 ${ }^{[46-48]}$ 。图 17 为文献[49-50]利用磁 悬浮磁阿基米德原理, 将踠豆种子和绿豆种子在 $\mathrm{GdCL}_{3}$ 水溶液(摩尔浓度为 $0.8049 \mathrm{~mol} / \mathrm{L}$ )中悬浮。

2004 年，美国 LYUKSYUTOV 等 ${ }^{[51]}$ 研制了基 于抗磁特性的微流控芯片, 实现了对微液滴、微粒 子的高精度操纵。其研制的芯片如图 18 所示, 两块

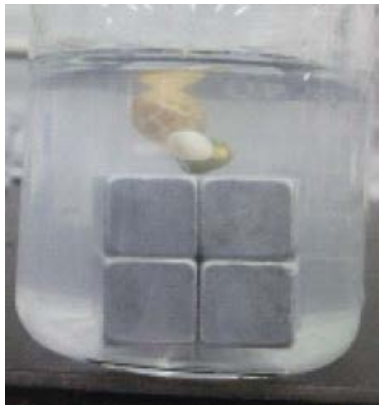

图 17 抗磁悬浮䇋豆种子和绿豆种子

永磁体反向排布在基板上, 微液滴/粒子抗磁悬浮在 两永磁体之间。基板上阵列了电极, 通过脉冲电极 产生的磁场实现操纵微液滴/粒子。

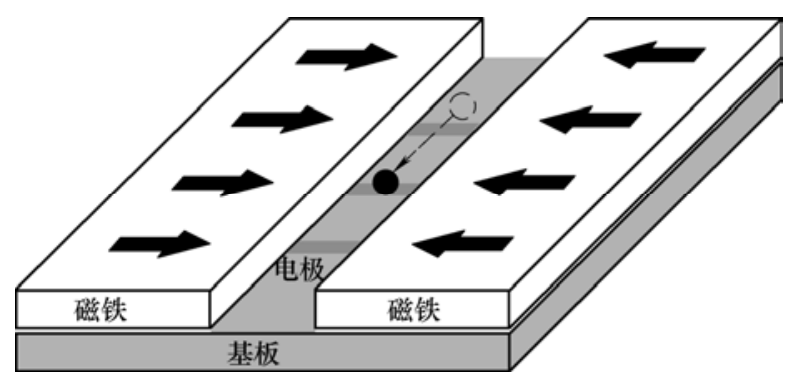

图 18 基于抗磁效应的微操纵芯片

CHETOUANI 等 ${ }^{[52]}$ 利用电镀制作工艺, 在硅基 底上溅射一层金作导电层, 甩光刻胶, 图形化, 然 后电镀 CoPt 层, 最后去除光刻胶得到微小永磁体阵 列。利用研制的微小永磁体阵列, 实现了对颗粒和 液滴非接触式操纵。PIGOT 等 ${ }^{[53]}$ 利用 MEMS 工艺 研制了微小 $\mathrm{NdFeB}$ 永磁体阵列, 实现了对金属浮铋 颗粒的悬浮(图 19)。

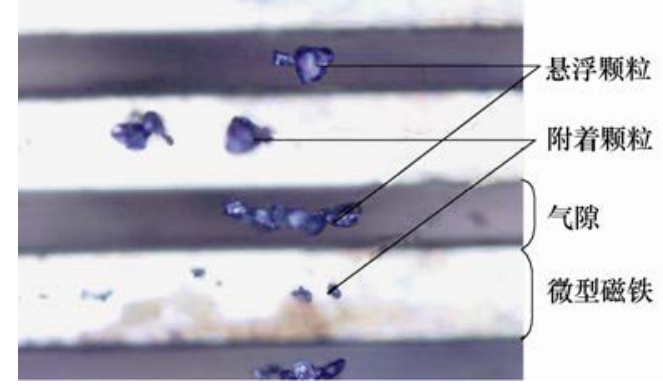

图 19 金属浮铋颗粒的悬浮

PEYMAN 等 ${ }^{[54]}$ 等针对微流控芯片中对微粒子、 细胞操纵需求, 利用抗磁效应实现了对微粒子捕获、 聚集和偏移三个方面的操纵, 如图 20 所示。

抗磁生物细胞/微粒子操纵技术无需强磁场环 境即可在常温下对活体生物粒子实现非接触、无损 伤的操纵, 已吸引了众多的研究人员的关注。除此 之外, 相关研究人员还根据抗磁物质的磁化率和密 度的不同, 实现对不同细胞的非接触分离 ${ }^{[55-56]}$, 进 一步提高了该技术的实用性。 


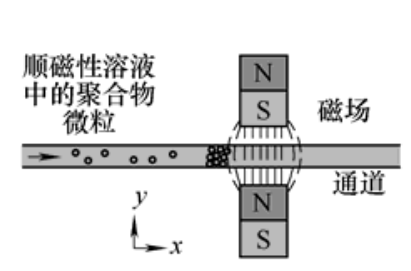

(a) 微粒子捕获

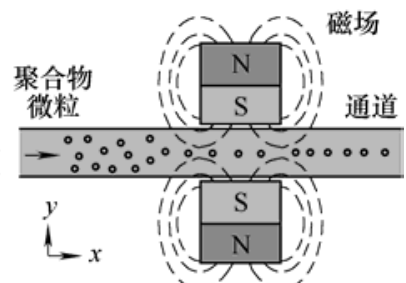

(b) 微粒子聚集

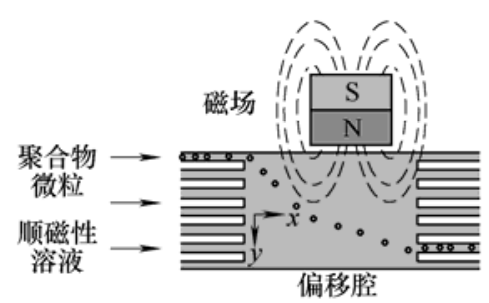

(c) 微粒子偏移

图 20 基于抗磁效应微粒子、细胞操纵

\section{4 研究展望}

抗磁悬浮技术经过近 30 年的发展, 已经取得了 丰硕的成果, 但弱抗磁效应始终是抑制抗磁悬浮技 术发展的瓶颈。因此, 抗磁悬浮未来发展的方向将 会侧重以下方面。

（1）基于抗磁悬浮的微纳器件研究。虽然在超 强磁场的作用下, 可以产生较大的抗磁力, 但这种 极为耗能的方式并不实用。如上文所述, 抗磁力与 物质重力之比将随着系统整体尺寸的减小而急剧增 大, 因而抗磁悬浮原理适宜于微器件中。现有的研 究成果大多集中于毫米量级及以上的宏观领域, 在 微纳米尺寸研究较少。未来抗磁悬浮微纳器件的研 究将会侧重于微纳永磁体阵列制作工艺、永磁体排 布的优化 ${ }^{[57]}$, 面向生命领域的基于抗磁效应的微流 控芯片、磁生物细胞/微粒子操纵、分离, 面向旋转 机械的高速微纳米电动机等。

(2) 抗磁性物质研究。抗磁性物质的磁化率是 抗磁效应强弱的决定因素。现有发现的物质在常温 下抗磁效应都很微弱, 若能提高抗磁物质的磁化率, 将会增强抗磁效应。因此, 在化学及材料领域研制 具有更强磁化率的抗磁性物质是未来抗磁悬浮技术 发展的趋势。

(3) 低频高灵敏度传感器。无摩擦、微弱的抗 磁悬浮支承刚度, 使得基于抗磁悬浮的传感器对低 频振动具有先天的高灵敏度特点。这个方向现有的 研究相对较少, 未来具有进一步研究的空间。

\section{5 结论}

（1）随着微细制造与强磁场技术的发展, 抗磁
悬浮相关研究开始兴起。针对国内对抗磁悬浮研究 相对较少的现状, 本文从抗磁悬浮原理、抗磁悬浮 特点及相关抗磁悬浮应用研究进行了详细综述。

（2）首先追述了物质的抗磁性被发现的历史及基 于抗磁特性相关经典研究成果。其次详述了物质的抗 磁效应、实现抗磁悬浮的条件及抗磁悬浮的特点。最 后本文详细归纳了抗磁悬浮在微纳旋转机械、振动能 量捕获、高灵敏度传感器、生物细胞/微粒子操纵等领 域的典型及最新研究成果, 并介绍了作者所在研究组 在抗磁悬浮在微纳旋转机械方面的研究成果。

(3) 为国内开展相关抗磁悬浮研究提供了综述 资料。

\section{参 考 文 献}

[1] KÜSTLER G. Diamagnetic levitation-historical milestones[J]. Revue Roumaine Des Sciences Techniques Serie Electrotechnique Et Energetique，2007， 52(3): 265-282.

[2] THOMSON W. On the forces experienced by small spheres under magnetic influence; and on some of the phenomena presented by diamagnetic substances[J]. Cambridge and Dublin Mathematical Journal, 1847, 5-6: 230-235.

[3] BERRY M V, GEIM A K. Of flying frogs and levitrons[J]. European Journal of Physics, 1997, 18(4): 307-313.

[4] SIMON M D, GEIM A K. Diamagnetic levitation: flying frogs and floating magnets[J]. Journal of Applied Physics, 2000, 87(9): 6200-6204.

[5] GEIM A K, SIMON M D, BOAMFA M I, et al. Magnet levitation at your fingertips[J]. Nature, 1999, 400 : 323-324.

[6] EARNSHAW S. On the nature of the molecular forces which regulate the constitution of the luminiferous ether[J]. Transactions of the Cambridge Philosophical Society, 1842, 7: 97-112.

[7] PELRINE R E. Room temperature, open-loop levitation of microdevices using diamagnetic materials[C]//IEEE Proceedings on Micro Electro Mechanical Systems, An Investigation of Micro Structures, Sensors, Actuators, Machines and Robots, 1990: 34-37.

[8] SIMON M D, HEFLINGER L O, GEIM A K. Diamagnetically stabilized magnet levitation[J]. American Journal of Physics, 2001, 69(6): 702-713.

[9] FURLANI E P. Permanent magnet and electromechanical devices: materials, analysis, and applications[M]. San Diego: Academic Press, 2001.

[10] PASQUALE G D， IAMONI S， SOMA A. 3D numerical modeling and experimental validation of diamagnetic levitating suspension in the static field[J]. International Journal of Mechanical Sciences, 2013, 68: 56-66. 
[11] SCHWEITZER G, MASLEN E H. Magnetic bearings: theory, design, and application to rotating machinery[M]. New York: Springer, 2009.

[12] CUGAT O, DELAMARE J, REYNE G. Magnetic micro-actuators and systems (MAGMAS)[J]. IEEE Transactions on Magnetics, 2003, 39(6): 3607-3612.

[13] CANSIZ A, HULL J R. Stable load-carrying and rotational loss characteristics of diamagnetic bearings[J]. IEEE Transactions on Magnetics, 2004, 40(3): 1636-1641.

[14] CANSIZ A. Static and dynamic analysis of a diamagnetic bearing system[J]. Journal of Applied Physics, 2008, 103(3): 034510.

[15] SU Y, XIAO Z, YE Z, et al. Micromachined graphite rotor based on diamagnetic levitation[J]. IEEE Electron Device Letters, 2015, 36(4): 393-395.

[16] 苏宇锋, 叶志通, 张坤. 抗磁悬浮石墨转子理论分析与 试验 $[\mathrm{J}]$. 中国机械工程, 2017，28(9): 1039-1043.

SU Yufeng, YE Zhitong, ZHANG Kun. Theoretical analyses and experiment of micromachined graphite rotor based on diamagnetic levitation[J]. China Mechanical Engineering, 2017, 28(9): 1039-1043.

[17] CHEN J Y, ZHOU J B, MENG G. Diamagnetic bearings for MEMS : Performance and stability analysis[J]. Mechanics Research Communications, 2008, 35(8): 546-552.

[18] CHEN J Y, ZHOU J B, MENG G, et al. Evaluation of eddy-current effects on diamagnetic bearings for microsystems[J]. IEEE Transactions on Industrial Electronics, 2009, 56(4): 964-972.

[19] MOSER R, BLEULER H. Precise positioning using electrostatic glass motor with diamagnetically suspended rotor[J]. IEEE Transactions on Applied Superconductivity, 2002, 12(1): 937-939.

[20] LIU W, CHEN W, ZHANG W, et al. Variable-capacitance micromotor with levitated diamagnetic rotor[J]. Electronics Letters， 2008，44(11): 681-683.

[21] 刘武. 抗磁悬浮转子微器件相关技术研究 [D]. 上海: 上 海交通大学, 2008.

LIU Wu. Related techniques of micromachined device with diamagnetic levitation rotor[D]. Shanghai: Shanghai Jiao Tong University, 2008.

[22] LIU K, ZHANG W, LIU W, et al. An innovative micro-diamagnetic levitation system with coils applied in micro-gyroscope[J]. Microsystem Technologies, 2010, 16(3): 431-439.

[23] XU Y, CUI Q, KAN R, et al. Realization of a diamagnetically levitating rotor driven by electrostatic field[J]. IEEE/ASME Transactions on Mechatronics, 2017, 22(5): 2387-2391.
[24] CIRRAN J, HUSSMAN C. Pioneering the application of diamagnetic materials for spacecraft attitude control[J]. IEEE Communications Magazine, 2015, 53(5): 200-201.

[25] LIU L, YUAN F. Nonlinear vibration energy harvester using diamagnetic levitation[J]. Applied Physics Letters, 2011, 98(20): 203507.

[26] LIU L, YUAN F. Diamagnetic levitation for nonlinear vibration energy harvesting: theoretical modeling and analysis[J]. Journal of Sound and Vibration, 2013, 332(2): 455-464.

[27] WANG X, PALAGUMMI S, LIU L, et al. A magnetically levitated vibration energy harvester[J]. Smart Materials and Structures, 2013，22(5): 055016.

[28] PALAGUMMI S, YUAN F. An optimal design of a mono-stable vertical diamagnetic levitation based electromagnetic vibration energy harvester[J]. Journal of Sound and Vibration, 2015, 342: 330-345.

[29] PALAGUMMI S, YUAN F. An enhanced performance of a horizontal diamagnetic levitation mechanism-based vibration energy harvester for low frequency applications[J]. Journal of Intelligent Material Systems and Structures, 2017，28(5): 578-594

[30] GAO Q, ZHANG W, ZOU H, et al. Design and analysis of a bistable vibration energy harvester using diamagnetic levitation mechanism[J]. IEEE Transactions on Magnetics, 2017, 53(10): 1-9.

[31] PASQUALE G D, SIYAMBALAPITIYA C, IAMONI S, et al. Characterization of low-stiffness suspensions based on diamagnetic levitation for MEMS energy harvesters[C]// Proc. Power MEMS, Leuven, Belgium, 2010: 77-80.

[32] 苏六帅, 叶志通, 苏宇锋. 微型抗磁悬浮振动能量采集 器输出特性分析[J]. 仪表技术与传感器, 2015(4): 46-48. SU Liushuai, YE Zhitong, SU Yufeng. Output performance analysis of micro-vibration energy harvester based on diamagnetic levitation[J]. Instrument Technique and Sensor, 2015(4): 46-48.

[33] 秦立振, 苏宇锋, 刘武发. 抗磁悬浮能量采集器结构参 数对输出特性影响 $[\mathrm{J}]$. 电子测量与仪器学报, 2016, 30(9): 1438-1443.

QIN Lizhen, SU Yufeng, LIU Wufa. Influence of structure parameter of diamagnetic levitation energy harvester on output performance[J]. Journal of Electronic Measurement and Instrumentation，2016，30(9): 1438-1443.

[34] 苏宇锋, 苏六帅. 微型抗磁悬浮振动能量采集器结构研 究与分析[J]. 机械设计与制造，2014(6): 84-86, 90. SU Yufeng, SU Liushuai. Research of micro-vibration energy harvester structure using diamagnetic levitation MAXWELL[J]. Machinery Design \& Manufacture, 
2014(6): 84-86, 90.

[35] KOBAYASHI M, ABE J. Optical motion control of maglev graphite[J]. Journal of the American Chemical Society, 2012, 134(51): 20593-20596.

[36] SIMON I, EMSLIE A G, STRONG P F, et al. Sensitive tiltmeter utilizing a diamagnetic suspension[J]. Review of Scientific Instruments， 1968，39(11): 1666-1671.

[37] GEIM A K, TISHA H A. Detection of earth rotation with a diamagnetically levitating gyroscope[J]. Physica B : Condensed Matter, 2001, 294: 736-739.

[38] BOUKALLEL M, PIAT E, ABADIE J. Passive diamagnetic levitation : theoretical foundations and application to the design of a micro-nano force sensor[C]//International Conference on Intelligent Robots and Systems, Las Vegas: IEEE/RSJ, 2003: 1062-1067.

[39] BILLOT M, PIAT E, ABADIE J, et al. External mechanical disturbances compensation with a passive differential measurement principle in nanoforce sensing using diamagnetic levitation[J]. Sensors and Actuators A: Physical, 2016, 238: 266-275.

[40] SHIMOKAWA Y, MATSUURA Y, HIRANO T, et al. Gas viscosity measurement with diamagnetic-levitation viscometer based on electromagnetically spinning system[J]. Review of Scientific Instruments, 2016, 87(12): 125105.

[41] GUNAWAN O, VIRGUS Y, TAI K. A parallel dipole line system[J]. Applied Physics Letter, 2015， 106: 062407.

[42] CAO Q, ZHANG W, ZOU H, et al. Tunable rotatingmode density measurement using magnetic levitation[J]. Applied Physics Letters, 2018, 112(14): 142408.

[43] NEMIROSKI A, SOH S, KWOK S W, et al. Tilted magnetic levitation enables measurement of the complete range of densities of materials with low magnetic permeability[J]. Journal of the American Chemical Society, 2016, 138(4): 1252-1257.

[44] 张承谦, 赵朋, 颉俊, 等. 抗磁性高密度物质的磁悬浮 密度测量方法[J]. 浙江大学学报, 2018, 52(4): 613-618. ZHANG Chengqian, ZHAO Peng, XIE Jun, et al. Density measurement by magnetic levitation for diamagnetic high-density materials[J]. Journal of Zhejiang University, 2018, 52(4): 613-618.

[45] IKEZOE Y, HIROTA N, NAKAGAWA J, et al. Making water levitate[J]. Nature, 1998, 393(6687): 749-750.

[46] IKEZOE Y, KAIHATSU T, SAKAE S, et al. Separation of feeble magnetic particles with magneto-Archimedes levitation[J]. Energy Conversion and Management, 2002, 43(3): 417-425.

[47] HIROTA N, KURASHIGE M, IWASAKA M, et al. Magneto-Archimedes separation and its application to the separation of biological materials[J]. Physica B, 2004, 346: 267-271.

[48] YOKOYAMA K, HIROTA N, IWASAKA M. Separation of collagen by magneto-Archimedes levitation[J]. IEEE Transactions on Applied Superconductivity, 2007, 17(2): 2181-2184.

[49] 李世鹏, 张卫平, 陈文元, 等. 抗磁悬浮的原理、特点 和应用 $[J]$. 磁性材料及器件, 2011，42(1)：1-6.

LI Shipeng, ZHANG Weiping, CHEN Wenyuan, et al. Principle, features and applications of diamagnetic levitation[J]. Journal of Magnetic Materials and Devices, 2011, 42(1): 1-6.

[50] 李世鹏. 基于 MEMS 的抗磁微操纵研究[D]. 上海: 上 海交通大学，2011.

LI Shipeng. Research of anti-magnetic micromanipulation base on MEMS[D]. Shanghai: Shanghai Jiao Tong University, 2011.

[51] LYUKSYUTOV I F，NAUGLE D G，RATHNAYAKA K D. On-chip manipulation of levitated femtodroplets[J]. Applied Physics Letters, 2004, 85(10): 1817-1819.

[52] CHETOUANI H, HAGUET V, JEANDEY C, et al. Diamagnetic levitation of beads and cells above permanent magnets[J]. Transducers, 2007, 2(5): 715-718.

[53] PIGOT C, CHETOUANI H, POULIN G, et al. Diamagnetic levitation of solids at microscale[J]. IEEE Transactions on Magnetics, 2008, 44(11): 4521-4524.

[54] PEYMAN S A, KWAN E Y, MARGARSON O, et al. Diamagnetic repulsion: A versatile tool for label-free particle handling in microfluidic devices[J]. Journal of Chromatography A, 2009, 1216(52): 9055-9062.

[55] INGLIS D W, RIEHN R, STURM J C, et al. Microfluidic high gradient magnetic cell separation[J]. Journal of Applied Physics, 2006, 99(8): 08K101.

[56] FURLANI E P. Magnetophoretic separation of blood cells at the microscale[J]. Journal of Physics: Applied Physics, 2007, 40: 1313-1319.

[57] KUZNETSOV S, GUETS J K. Topology optimization of magnetic source distributions for diamagnetic and superconducting levitation[J]. Journal of Magnetism and Magnetic Materials, 2017, 438: 60-69.

作者简介: 徐园平, 男, 1989 年出生, 博士。主要研究方向为磁悬浮技术。 E-mail: ypxu@nuaa.edu.cn

周瑾(通信作者), 女, 1972 年出生, 博士, 教授, 博士研究生导师。主 要研究方向为磁悬浮技术、转子动力学、机电系统控制。

E-mail: zhj@nuaa.edu.cn

金超武, 男, 1980 年出生, 博士, 副教授, 硕士研究生导师。主要研究 方向为磁悬浮技术。

E-mail: jinchaowu@nuaa.edu.cn

BLEULER Hannes, 男, 1954 年出生, 博士, 瑞士洛桑联邦理工学院终 身教授, 博士研究生导师。主要研究方向为磁悬浮技术、医疗机器人。

E-mail: hannes.bleuler@epfl.ch 